\title{
Study of the Construction of a Coastal Disaster Prevention System using Deep Learning
}

\author{
Yeon-Joong Kim $\circledast^{*}$, Tae-Woo Kim $\circledast^{*}$, Jong-Sung Yoon $\circledast^{*}$ and Myong-Kyu Kim $\circledast^{* *}$ \\ "Department of Civil and Urban Engineering Inje University, Gimhae, Korea \\ ${ }^{*}$ Hydro Technology Institute Co. Ltd., Busan, Korea

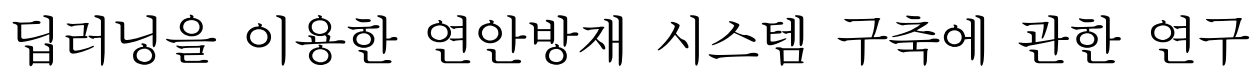

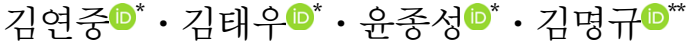 \\ *인제대학교 토목도시공학부 \\ *(주)HTI Korea R\&D Center
}

KEY WORDS: Disaster prevention system 방재 시스템, Artificial intelligence 인공지능, Deep learning 딥러닝, Big data 빅데이터

\begin{abstract}
Numerous deaths and substantial property damage have occurred recently due to frequent disasters of the highest intensity according to the abnormal climate, which is caused by various problems, such as global warming, all over the world. Such large-scale disasters have become an international issue and have made people aware of the disasters so they can implement disaster-prevention measures. Extensive information on disaster prevention actively has been announced publicly to support the natural disaster reduction measures throughout the world. In Japan, diverse developmental studies on disaster prevention systems, which support hazard map development and flood control activity, have been conducted vigorously to estimate external forces according to design frequencies as well as expected maximum frequencies from a variety of areas, such as rivers, coasts, and ports based on broad disaster prevention data obtained from several huge disasters. However, the current reduction measures alone are not sufficiently effective due to the change of the paradigms of the current disasters. Therefore, in order to obtain the synergy effect of reduction measures, a study of the establishment of an integrated system is required to improve the various disaster prevention technologies and the current disaster prevention system. In order to develop a similar typhoon search system and establish a disaster prevention infrastructure, in this study, techniques will be developed that can be used to forecast typhoons before they strike by using artificial intelligence (AI) technology and offer primary disaster prevention information according to the direction of the typhoon. The main function of this model is to predict the most similar typhoon among the existing typhoons by utilizing the major typhoon information, such as course, central pressure, and speed, before the typhoon directly impacts South Korea. This model is equipped with a combination of AI and DNN forecasts of typhoons that change from moment to moment in order to efficiently forecast a current typhoon based on similar typhoons in the past. Thus, the result of a similar typhoon search showed that the quality of prediction was higher with the grid size of one degree rather than two degrees in latitude and longitude.
\end{abstract}

\section{1. 서 론}

최근 세계적으로 지구 온난화 등의 여러 문제로 인한 이상기 후에 따른 최대급 강도의 재해가 빈번히 발생되어 수많은 인명 및 재산피해가 발생되고 있다. 이러한 대규모 재해는 국제적인 이슈가 되어 재난에 대한 경각심과 그에 따른 방재대책의 관심 도 고조되고 있다. 2018년 8월에 발생한 슈퍼태풍 21호 제비가 일본에 상륙하여 일본 킨키 지방을 중심으로 많은 피해가 발생 하였다. 직접 영향권인 오사카에서는 관측(9월 4일) 이래 최대
조위가 기록되었고 태풍의 간접 영향권에 있던 북해도에서도 집중강우가 발생되었다. 이후 북해도에서는 강우에 의해 연약 해진 지반상태에서 9월 6일 진도 7의 강진이 발생하여 수십명 의 인명피해와 사회적 기능이 마비되는 등 복합적인 피해가 발 생하였다. 이와 같이 동시 다발적으로 발생되는 자연 재해에 맞 서 정부 및 지방자치단체는 방재 기술력 향상에 많은 연구를 진행하고 있지만 현재 발생되는 재해 특성상 예측이 난해해 방 재대책 구축에 많은 난항을 겪고 있다.

우리나라와 매우 인접하게 위치하고 있는 일본에서는 특히

Received 4 August 2019, revised 6 November 2019, accepted 14 November 2019

Corresponding author Myoung-kyu Kim: +81-80-3825-9086, k98514007@gmail.com ORCID: http://orcid.org/0000-0002-0639-7577 It is noted that this paper is revised edition based on proceedings of KSCDP 2019 in Jeju.

(C) 2019, The Korean Society of Ocean Engineers

This is an open access article distributed under the terms of the creative commons attribution non-commercial license (http://creativecommons.org/licenses/by-nc/3.0) which permits unrestricted non-commercial use, distribution, and reproduction in any medium, provided the original work is properly cited. 
2011년 3월 동일본 대지진 재해와 같은 최대급 규모의 재해가 발생하였고 이후 지속적으로 여러 자연재해가 발생되고 있다. 뿐만 아니라, 향후 30 년 이내에 난카이 토라후 지진(대규모 지 진)이 발생할 확률을 $70 \%$ 이상으로 예측(예상 피해액 약 2,100 조 앤, 일본 토목학회)되고 있어 장기 지속적으로 철저한 준비와 대비를 하고 있다. 하지만 대규모 재해에 대한 발생 자체의 억 제와 정확한 예측은 불가능하다. 따라서 산재된 방재 시스템을 방재 목적에 부합되도록 통합 관리하고 이때 불충분한 점은 보 완 및 개발을 통해 방재 인프라를 조기에 구축하여 가능한 피 해를 최소화하기 위한 노력이 현재의 방재 시스템 구축에 있어 매우 중요하다.

세계적으로 자연재해 저감 대책을 지원하기 위한 많은 방재 정보가 적극적으로 공표되고 있다. 일본의 경우 여러 대규모 재 해로 부터 얻은 방대한 방재 데이터를 바탕으로 하천, 해안-항 만 등 여러 분야에서 계획빈도 뿐 아니라 예상최대빈도에 따른 외력을 산정하여 해저드 맵 구축(Kim et al., 2015) 및 수방활동 을 지원하는 다양한 방재 시스템(Sugiura et al., 2015)의 개발 연 구도 활발히 진행되고 있다. 하지만 현재 발생되는 재해의 패러 다임이 변화하고 있어 현행의 저감 대책만으로 큰 효과를 기대 하기가 매우 어려운 상황이다. 따라서 저감대책의 시너지 효과 를 얻기 위해서는 현행의 방재 시스템의 개량과 더불어 다방면 의 방재 기술력을 향상시켜 통합적인 시스템 구축에 관한 연구 가 필요하다.

본 연구에서는 방재 인프라 구축 및 확장을 위한 연구로서 연 안방재 시스템 개발을 위해 태풍이 상륙하기 전의 태풍 정보를 이용하여 지금까지 발생된 태풍 중 가장 유사한 태풍을 인공지 능(Artificial intelligence, $\mathrm{AI}$ )을 이용해 예측하고 태풍이 진행함 (상륙)에 따른 주요한 방재정보를 제공하여 수방활동에 필요한 정보 제공 및 피해저감을 목적으로 한다. 또한 선행 연구인 뉴 럴 네트워크의 최적화에 검토(Kim et al., 2019)에 따라 연안방 재 시스템의 성능 및 신뢰성 향상을 위한 시스템 구축을 검토 한다. 모델의 주요한 기능으로는 태풍이 우리나라에 직접적인 영향을 미치기 전, 태풍의 주요 태풍정보인 경로, 중심기압, 이 동속도를 이용해 기존에 발생된 태풍 중 가장 유사한 태풍을 예측한다. 이때 과거 발생된 유사 태풍의 효율적인 예측을 위해 인공지능(AI)을 이용하여 심층학습(Deep learning)을 적용한 심 층 신경망(Deep neural network, DNN) 기술에 따라 시시각각 변 화하는 태풍을 예측하였다. 모델의 구축은 선행 연구에 의해 구 축한 알고리즘을 사용하며 격자 크기와 학습 데이터의 선형보 간에 따른 민감도 분석을 실시하였다. 그 결과 학습 데이터의 구축에 따라 예측 적중률이 크게 향상되었다.

\section{2. 심층학습(Deep learning)}

기계학습(Machine learning)의 알고리즘은 수집된 데이터로부 터 법칙성을 찾아내는 방법이며 인공지능의 기술을 기반으로 하고 있다. 그 중에서도 최근 심층학습을 이용한 인공 뉴럴 네 트워크(Artificial neural network, ANN)의 발전이 많은 연구자를 통해 여러 분야에서 활용되고 있다. 현재 방재 분야에서는 주로 지역적인 특성을 최대한 반영하여 물리모델을 구축하고 이를
통해 예측 결과를 산출해 방재활동에 중요한 정보로 활용되고 있다. 일본에서는 과거부터 수많은 방재 데이터를 축적하고 있 어 기계학습에 있어 많은 장점을 활용해 여러 분야에 걸쳐 연 구가 진행 중에 있다. 특히 심층학습을 이용한 하천-수위 예측 수법의 개발(Hitokoto et al., 2016), 심층학습을 이용한 감조하천 의 수질변동 예측(Nakatani et al., 2017) 등과 같이 비교적 물리 모델을 구축보다는 비교적 용이하게 모델을 구축하여 좋은 결 과를 제시하고 있다. 우리나라에서도 여러 분야에서 정도 좋은 데이터가 축적되어 이를 활용해 기계학습에 대한 연구가 활발 히 진행되고 있다.

\section{1 심층학습을 이용한 심층 신경망 모델}

심층 신경망(DNN)은 입력층(Input layer)과 출력층(Output layer)사이에 여러 개의 은닉층(Hidden layer)들로 이뤄진 인공 신경망(ANN)이다. 심층 신경망은 일반적인 인공 신경망과 마찬 가지로 복잡한 비선형 관계(Non-linear relationship)의 모델링이 가능하다. 일반적으로 은닉층을 2 층 이상의 뉴럴 네트워크를 심 층학습이라 하며 Fig. 1에 일반적인 뉴럴 네트워크의 구성도를 나타내다. 본 연구에서는 선행 연구(Kim et al., 2019)를 바탕으 로 모델을 구축하였으며 은닉층은 2층으로 심층 신경망(DNN) 을 구축하였다. 【 Simple Neural Wetwork】【 Deep Learning Neural Network】
input hidden output input hidden1 hidden2 output
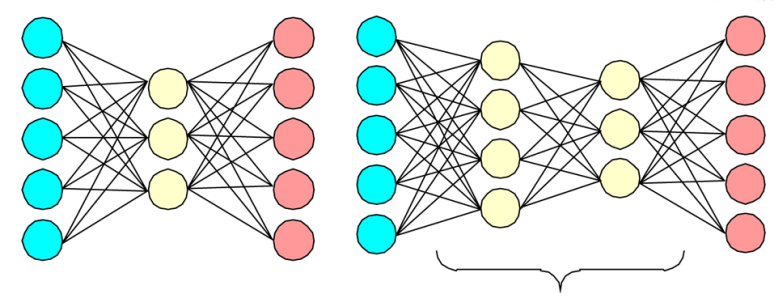

hidden layer $=2$

Fig. 1 Structures of neural network

\subsection{Neural Network의 학습방법}

활성화 함수(Activation function)는 인공지능의 많은 알고리즘 에서 다양한 형태로 사용되고 있다. 뉴럴 네트워크는 신경세포 의 구조를 모방한 수학모델이며 일정한 입력 데이터(Input layer) 에 반응하여 적합한 데이터를 출력(Output layer)하는 구조이다. Fig. 2에 뉴럴 네트워크의 은닉층의 구성을 나타냈다.

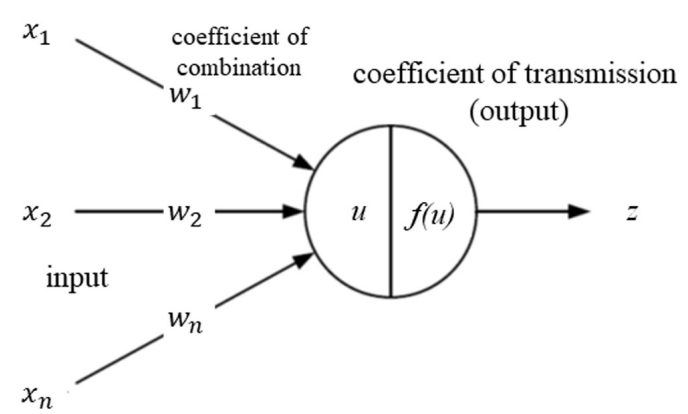

Fig. 2 Neural network diagram of element 


$$
\begin{aligned}
& u=\theta_{i}+\sum_{i=1}^{K} w_{i} x_{i} \\
& z=f(u)
\end{aligned}
$$

여기서 $X_{i}$ 는 입력층, $W_{i}$ 는 Weight(입력치의 중요성), $B$ 는 Bias (입력치의 총계를 조절하는 파라미터), $f(u)$ 는 활성화 함수 (Activation function), $Z$ 는 출력층이다. 신경망에서 사용되는 대 표적인 활성함 함수는 시그모이드(Sigmoid)형, 계단함수(Hard limiter), 임계논리(Threshold logic)함수, ReLU(Rectified linear unit) 등이 있으며, 본 연구에서는 격자상에 원활한 태풍경로를 학습시키기 위해 선행 연구에 의해 ReLU(Iteration number=300) 의 활성화 함수(식 (3))를 사용하여 모델을 구축하였다.

$$
f(u)=\max (0, u)
$$

일반적으로 은닉층의 개수가 증가함에 따라 더욱 많은 문제 를 해결 할 수 있도록 학습 능력이 향상되는 반면, 학습 데이터 의 과잉학습에 의해 실제 데이터에 대한 오차가 증가하는 현상 과적합(Overfitting)이 발생하여 예측값의 정도가 하락하는 문제 점이 발생한다. 따라서 은닉층의 개수에 따른 모델의 평가와 더

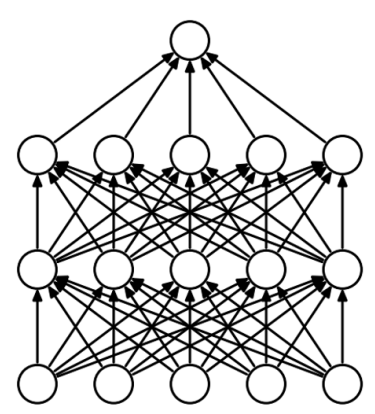

(a) Standard neural net

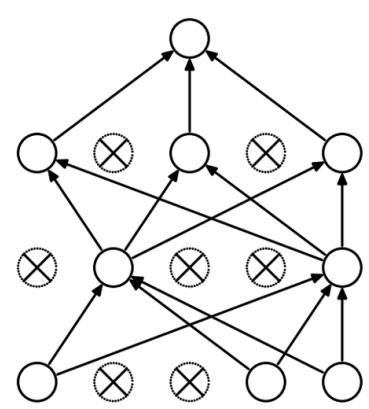

(b) After applying dropout
Fig. 3 Dropout neural net model

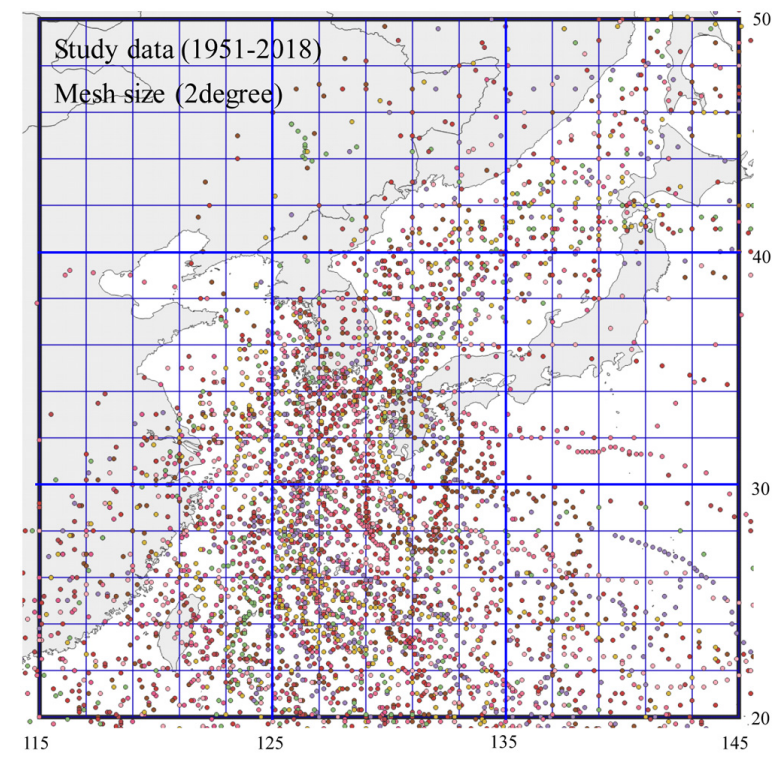

Fig. 5 Boundary condition

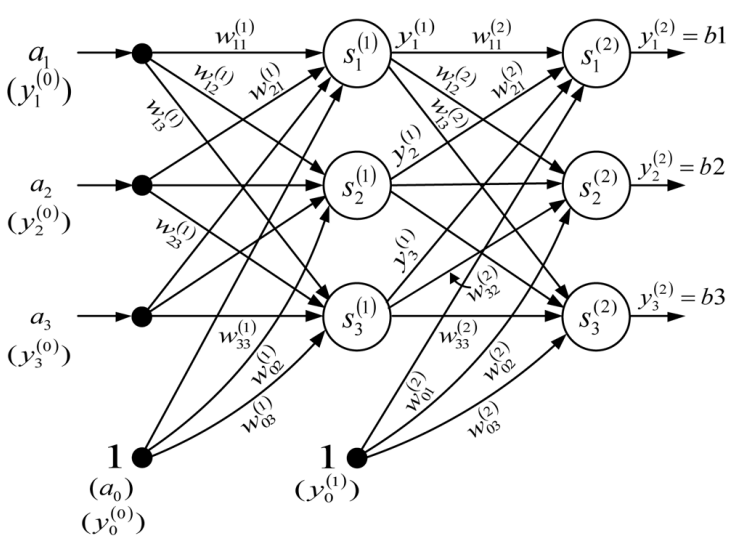

Fig. 4 Feed forward method

불어 심층학습에서 과적합을 감소시키는 방법으로(Fig. 3) 본 연 구에서는 Dropout(Srivastava et al., 2014)을 적용한다.

뉴럴 네트워크에 사용되는 주요 학습방법은 Hebbian Learning, Perceptron Rule, Gradient descent 및 오차역전파법(Back propagation) 등이 있다. 본 연구에서는 오차역전파법은 Feed forward 형(Fig. 4) 뉴럴 네트워크의 대표적인 학습법으로 훈련 데이터가 주어질 때마다 결선무게(Weigh on a synapse)를 미세 수정하는 방법을 적 용하였다.

\section{3. 유사태풍 예측 시스템 구축}

학습 데이터(Study data)인 태풍의 주요 정보인 이동경로, 중심 기압 및 이동속도에 대한 정보는 National Institute of Informatics (NII, 2019)에서 제공되는 Digital typhoon를 이용하였고 구축한 Mesh 정보를 Fig. 5 에 나타내었다. Mesh 데이터의 간격은 위경도 각각 $2^{\circ}$ 및 $1^{\circ}$ 로 구축하여 연속운동을 하는 태풍의 주요정보를 학 습하기 위해 태풍 정보를 1시간 간격 데이터로 선행보간 (Fig. 6) 하였다. Digital typhoon 데이터가 존재하는 태풍(1951 2018)을 대 상으로 태풍연구센터(Typhoon Research Center, 2019)에서 조사한

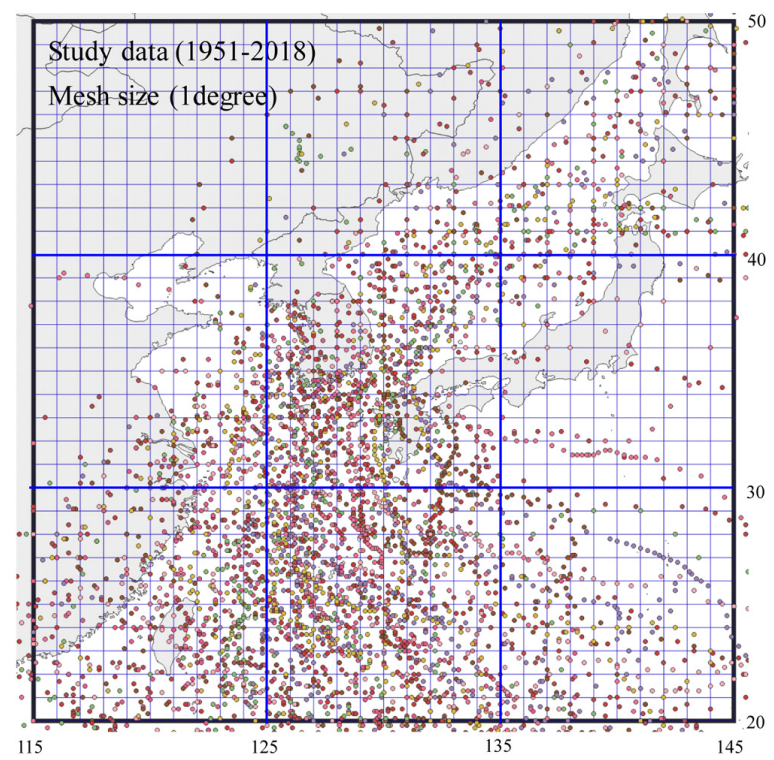




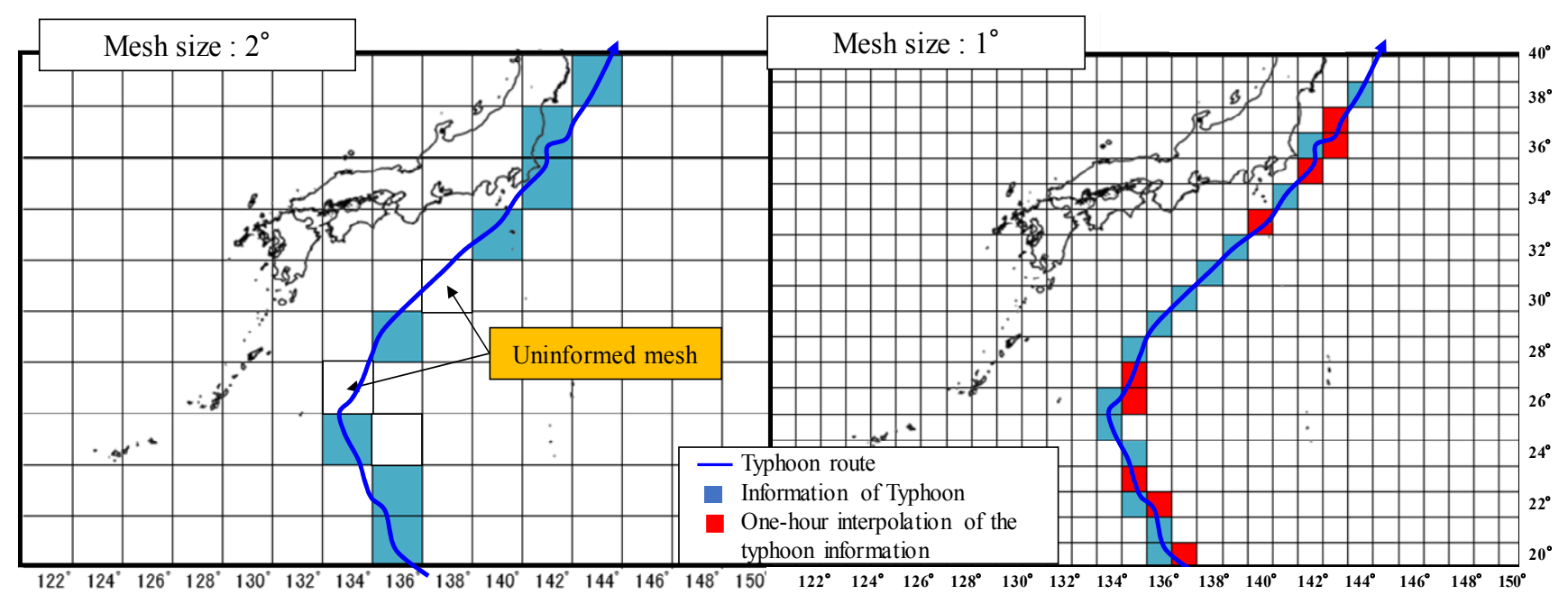

Fig. 6 Mesh segmentation in typhoon information with interpolation

Table 1 Parameters for Neural network

\begin{tabular}{|c|c|c|c|}
\hline Parameter & Case-I & Case-II & Case-III \\
\hline Model & \multicolumn{3}{|c|}{ Deep neural network (DNN) } \\
\hline Hidden layer & 2 layers & 2 layers & 2 layers \\
\hline $\begin{array}{l}\text { Activation } \\
\text { function }\end{array}$ & ReLU & ReLU & ReLU \\
\hline Dropout & O & O & $\bigcirc$ \\
\hline Mesh size & 2 degree & 2 degree & 1 degree \\
\hline Range & \multicolumn{3}{|c|}{$\begin{aligned} \text { latitude } & 20 \sim 50 \\
\text { longitude } & 115 \sim 145\end{aligned}$} \\
\hline Input parameter & \multicolumn{3}{|c|}{ Route, Pressure, Moving speed } \\
\hline $\begin{array}{l}\text { Interpolation } \\
(1 \text { hour })\end{array}$ & $x$ & $\bigcirc$ & $\bigcirc$ \\
\hline Input units & 675 & 675 & 2700 \\
\hline Middle units & $\begin{array}{l}1 \text { layer: } 675 \\
2 \text { layer: } 675\end{array}$ & $\begin{array}{l}1 \text { layer: } 675 \\
2 \text { layer: } 675\end{array}$ & $\begin{array}{l}1 \text { layer: } 2700 \\
2 \text { layer: } 2700\end{array}$ \\
\hline Output units & 214 & 214 & 214 \\
\hline Study data & \multicolumn{3}{|c|}{$214(1951 \sim 2017)$} \\
\hline Test data & \multicolumn{3}{|c|}{$4(2018)$} \\
\hline
\end{tabular}

우리나라에 영향을 끼친 태풍 218를 학습 및 Test 데이터를 구축 하였고 모델의 구성 및 검증 계산 조건을 Table 1 에 표시하였다.

최근 인공지능(AI) 개발에 적합한 많은 프로그래밍 언어(R, Python, JavaScript, $\mathrm{C} / \mathrm{C}+$, Matlab 등)가 개발되고 있으며 본 연 구에서는 $\mathrm{MxNet}$ 프레임워크를 이용해 $\mathrm{R}$ 의 언어를 이용해 모델 을 구축하였다. $\mathrm{R}$ 의 가장 큰 장점은 방대한 양의 패키지와 즉시 사용 가능한 테스트 세트가 제공되어 비교적 데이터의 구축, 모 델의 학습, 모델의 평가 등 모델의 구축이 용이하다.

학습 데이터의 주요 인자인 태풍의 중심기압, 이동속도에 대 해 관계형 데이터베이스의 중복을 최소화하기 식 (4)에 의해 정 규화(Normalization)과정을 거쳐 학습데이터를 구축하였다. 이때 태풍의 중심기압은 $900(=1.0) \sim 1000(=0.0) \mathrm{hPa}$ 의 분포로 설정하였 고 이동속도는 $0(=0.0) \sim 75(=1.0) \mathrm{m} / \mathrm{s}$ 의 정규화 분포로 설정하였 다. 또한 태풍의 이동 경로는 구축한 격자범위 안에 위치하고
있으면 1.0 이외는 0.0 으로 설정하였다.

$$
x_{n e w}=\frac{x-x_{\min }}{x_{\max }-x_{\min }}
$$

\section{4. 유사태풍 예측 결과}

선행 연구에서의 태풍정보 입력 Mesh의 크기가 위경도 $2^{\circ}$ (Without interpolation)를 사용하고 있어 비교적 Mesh의 크기에 따라 학습데이터의 중복학습으로 인한 유사태풍 예측의 정확도 가 감소되는 문제점을 보완하였다. Mesh 크기를 $2^{\circ}$ 에서 $1^{\circ}$ 크기 로 상세하게 구축하였으며, Digital typhoon의 주요 정보는 최대 6시간 간격으로 제공되고 있어 태풍의 이동속도에 따라 태풍정 보가 누락되어 태풍의 이동특성을 재현하지 못하는 한계점을 보완하기 위해 최소 1 시간 간격의 데이터를 선형적으로 보간하 여 학습데이터를 구축하였다. 모델의 검증을 위해 학습 데이터 (Study data)를 1951년 2017년에 발생된 태풍(214개)으로 구축하 였고 테스트 태풍은 2018년 발생한 우리나라에 영향이 미친 태 풍 4개(07호, 15호, 19호, 25호)를 대상으로 예측 모의를 실시하 였다. 모델의 예측 결과는 입력받은 값을 $0 \sim 1$ (정규화)사이의 값 으로 출력되는 값의 총합이 1 이 되는 특성을 가진 Softmax 함수 값으로 유사성을 평가하였고 인공지능을 이용한 예측 모델의 주요한 결과는 다음과 같다.

\section{1 학습데이터의 선형보간}

Fig. 6과 같이 태풍의 이동속도 및 제공되는 정보의 위치는 Mesh에서 연속적인 태풍의 위치를 표현하지 못하는 단점을 보 완하기 위해 최소 1 시간 간으로 선형보간 하여 태풍의 연속적 인 데이터를 제공하여 모델을 구축한 결과 선형보간을 하지 않 은 경우(Case-I)보다 선형보간을 한 경우(Case-II)의 적중률(Rank. 1)이 크게 증가하였다(Table 2). 또한 적중률 1위의 태풍경로의 비교(Fig. 7(a))에서도 격자를 선형보간 경우가 사람의 눈으로 판단했을 경우와 같이 보다 유사한 태풍이 예측되었고 Case-I과 Case-II의 결과에서는 Test 태풍 3개(07호, 15 호, 19호)의 예측 순 
위 1 위가 동일한 태풍으로 예측되었지만 적중률 값(Softmax)이 크게 증가함에 따라 예측 결과의 신뢰성을 확인하였다. 따라서 이 결과로부터 학습데이터의 선형보간에 따른 예측 성능의 향 상과 더불어 인공지능을 통한 예측에 있어 학습데이터 질과 양 에 크게 의존하며 양질의 학습데이터 구축이 매우 중요한 것으 로 판단된다.

\subsection{Mesh 크기에 따른 감도분석}

운동하는 태풍의 이동 특성을 반영하기 위한 학습 데이터의 구축을 위해 Mesh $2^{\circ}$ (Case-II)에서 $1^{\circ}$ (Case-III)로 보다 상세하게 모델의 격자정보를 구축하였으며 Mesh 크기의 축소와 함께 ‘ 4.1 학습데이터의 선형보간'의 결과에 따라 태풍의 정보를 선형보 간하여 학습데이터를 구축하였다. 그 결과 격자수의 증가(4배) 로 인해 적중률은 비록 미세하게 감소하였지만 Fig. 7(b)의 결과
와 같이 태풍의 이동경로에 대해서는 $1^{\circ}(\mathrm{Case}-\mathrm{III})$ 의 경우가 보 다 유사한 태풍을 예측되었다. 대체로 우리나라에 영향을 미치 는 태풍의 경로는 제주도를 포함해서 남해에서 동해(독도)로 이 동하는 태풍의 경로가 다수이므로 서로 중첩되는 태풍의 경로 가 지배적이다. 따라서 태풍의 경로를 예측하기 위해서는 각각 의 태풍정보가 중첩되는 것을 방지하기 위해 Mesh의 크기는 $2^{\circ}$ 보다 $1^{\circ}$ 의 경우가 적절한 것으로 판단된다.

\section{3 방재 시스템(Disaster prevention system)}

방재 시스템으로 활용하기 위해 구축한 모델을 이용하여 2017년에 발생한 태풍 21호 'Lan'(2017T21)을 대상으로 육지에 상륙 1,2 일전의 태풍 조건으로 각각의 유사태풍을 실시하였고 각각의 유사태풍 예측 결과 순위 1위를 Fig. 8에 나타내었다. 태 풍이 육지로 점차 근접함에 따라 시시각각 변화하는 태풍 정보

Table 2 Results of prediction model

\begin{tabular}{|c|c|c|c|c|c|c|c|c|c|c|c|c|}
\hline \multirow{2}{*}{ No. } & \multirow{2}{*}{$\begin{array}{c}\text { Typhoon } \\
\text { (Test) }\end{array}$} & \multicolumn{5}{|c|}{ Typhoon (Prediction) / Rank. } & \multicolumn{6}{|c|}{ Value of softmax } \\
\hline & & 1 & 2 & 3 & 4 & 5 & 1 & 2 & 3 & 4 & 5 & $\Sigma$ \\
\hline \multirow{4}{*}{ Case-I } & $2018 \mathrm{~T} 07$ & 197408 & 199109 & 199113 & 200613 & 199905 & 0.516 & 0.404 & 0.025 & 0.019 & 0.010 & 0.974 \\
\hline & $2018 \mathrm{~T} 15$ & 200610 & 196804 & 199211 & 197209 & 199407 & 0.662 & 0.110 & 0.046 & 0.043 & 0.042 & 0.902 \\
\hline & $2018 \mathrm{~T} 19$ & 198508 & 200215 & 201207 & 200603 & 197006 & 0.740 & 0.141 & 0.025 & 0.024 & 0.021 & 0.952 \\
\hline & $2018 \mathrm{~T} 25$ & 201618 & 200415 & 197408 & 198410 & 199711 & 0.330 & 0.320 & 0.172 & 0.124 & 0.023 & 0.969 \\
\hline \multirow{4}{*}{ Case-II } & 2018T07 & 197408 & 198410 & 196715 & 199905 & 198118 & 0.886 & 0.023 & 0.017 & 0.016 & 0.014 & 0.956 \\
\hline & $2018 \mathrm{~T} 15$ & 200610 & 196804 & 199211 & 195204 & 197209 & 0.737 & 0.128 & 0.036 & 0.014 & 0.014 & 0.929 \\
\hline & $2018 T 19$ & 198508 & 197119 & 196617 & 200603 & 197006 & 0.948 & 0.015 & 0.014 & 0.006 & 0.005 & 0.988 \\
\hline & $2018 \mathrm{~T} 25$ & 200415 & 199711 & 198410 & 197408 & 198118 & 0.542 & 0.373 & 0.052 & 0.008 & 0.006 & 0.981 \\
\hline \multirow{4}{*}{ Case-III } & 2018T07 & 197408 & 198410 & 195204 & 199109 & 196715 & 0.813 & 0.061 & 0.042 & 0.023 & 0.007 & 0.946 \\
\hline & $2018 \mathrm{~T} 15$ & 200610 & 197209 & 199211 & 199407 & 196804 & 0.490 & 0.147 & 0.135 & 0.050 & 0.028 & 0.850 \\
\hline & $2018 T 19$ & 198508 & 197119 & 196617 & 195209 & 196104 & 0.415 & 0.409 & 0.054 & 0.023 & 0.014 & 0.915 \\
\hline & $2018 \mathrm{~T} 25$ & 199711 & 200415 & 198410 & 197408 & 198118 & 0.548 & 0.373 & 0.038 & 0.014 & 0.007 & 0.981 \\
\hline
\end{tabular}

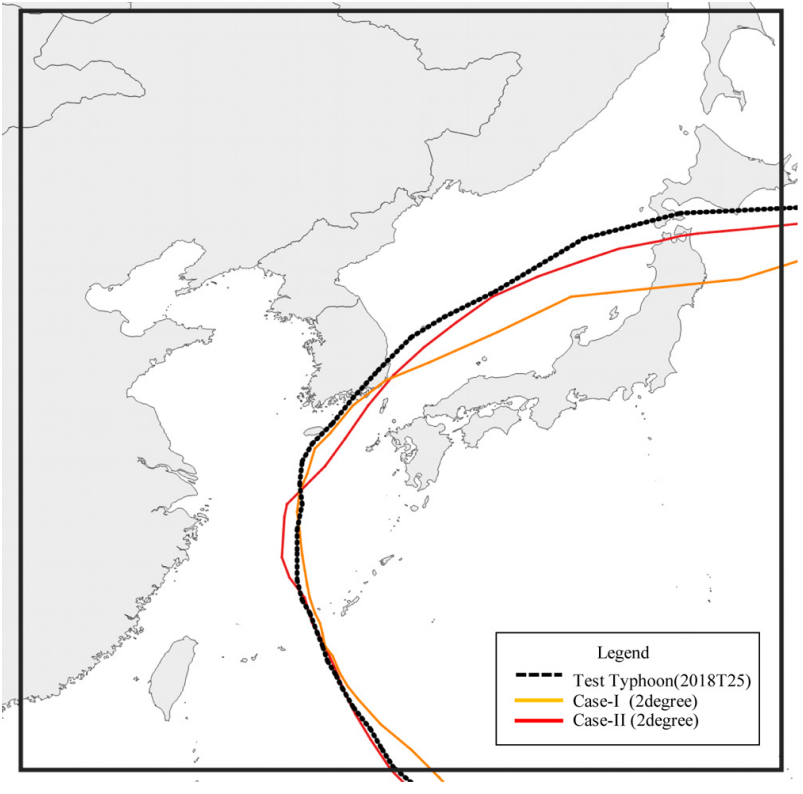

(a) Result by Interpolation

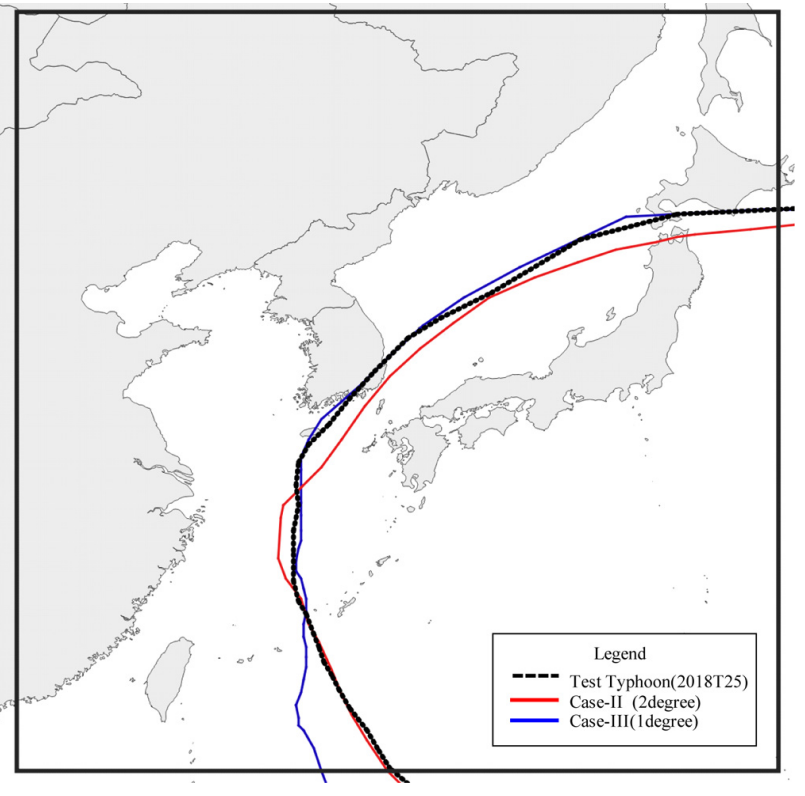

(b) Result by mesh size

Fig. 7 Results of similar typhoon for Kong-rey (2018T25) 


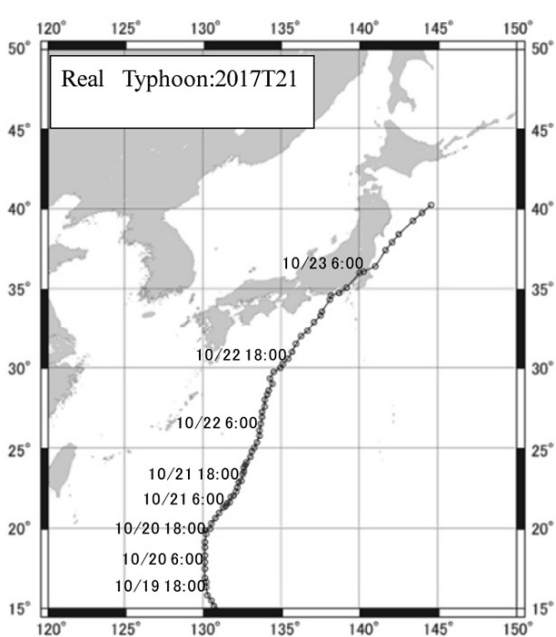

(a) Typhoon route $(2017 \mathrm{~T} 21)$

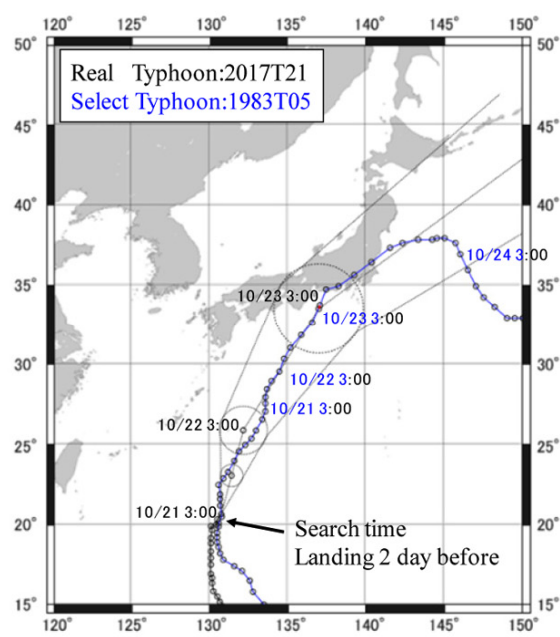

(b) Landing 2 day before

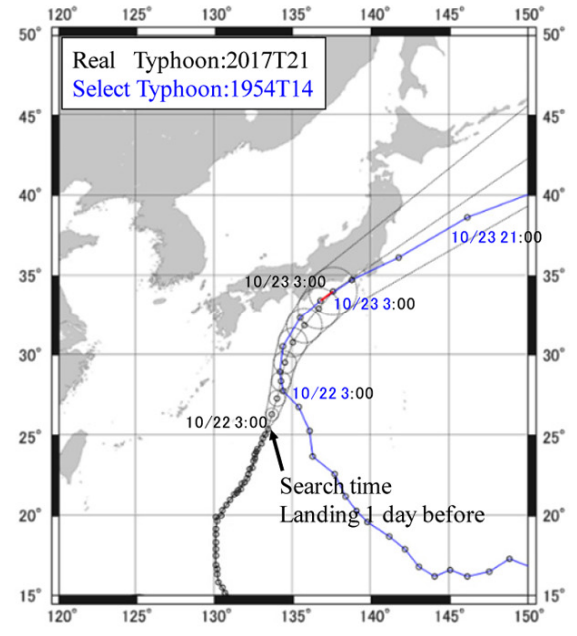

(c) Landing 1 day before

Fig. 8 Results of research typhoon due to search time

를 바탕으로 예측되는 태풍 또한 변화하는 것으로 나타났다. 이 결과로부터 태풍이 점차 근접함에 따라 수방체제로 접어드는 시기와 함께 유사태풍 예측 시스템의 결과로부터 얻어진 예측 태풍의 방재정보(예측된 유사 태풍에 의해 발생된 재해규모, 피 해범위, 주요 하천수위 등)를 미리 파악하여 피해 경감을 위한 주요한 방재 지원 시스템으로 활용이 가능한 것으로 나타났다.

\section{5. 결 론}

본 연구에서는 최대급 규모에 대한 피해저감을 위한 비구조 적 대책으로 연안방재 시스템 구축을 위한 모델의 재현정도 향 상 및 방재 시스템으로서의 활용가치 및 적용성을 평가하였다. 새롭게 구축한 모델의 기능을 유사태풍 검색에 초점을 맞춰 평 가한 결과, 검색 재현성이 크게 향상되었고 방재 시스템으로서 의 활용 가치를 확인하였다. 본 연구의 주요한 연구 결과는 다 음과 같다.

(1) 입력되는 태풍의 주요정보(Digital typhoon)를 최소 1시간 간격으로 선형적인 형태로 보간하고 태풍정보의 학습에 있어 중첩을 피하기 위해 격자 크기를 $1^{\circ}$ 로 구축하였다. 그 결과 학 습데이터의 선형보간으로 예측 적중률이 크게 향상되었고 격자 크기를 $2^{\circ}$ 의 학습데이터 보다 $1^{\circ}$ 의 경우가 보다 유사한 태풍을 예측되는 것으로 나타났다.

(2) 여러 방재 시스템과의 적용을 위해 구축한 모델을 이용해 실시간의 태풍정보(기상청 예측정보)를 이용하여 유사태풍을 예측하고 실제 발생된 태풍이 육지에 상륙하기 전에 예측된 과 거 태풍의 방재 정보(피해강도, 재해범위, 하천 수위 등의)를 미 리 파악하여 수방체제로 접어드는 시기와 함께 중요한 방재 정 보를 제공함으로서 방재 지원 시스템으로서의 활용 가치를 확 인하였다.

(3) 인공지능을 이용한 예측 분야에 있어 학습되는 데이터의 양과 질에 따라 그 예측 결과가 상이한 것으로 나타났으며 이 는 양질의 학습데이터 구축이 매우 중요한 것으로 판단된다.

향후의 연구로서 자연재해 저감대책을 지원하는 유사 태풍 검색 시스템을 바탕으로 과거에 발생한 태풍이 동반한 강우사
상에 의해 발생된 검토대상지역의 주요하천 수위를 산정하여 하천수위 정보를 제공함으로서 수방활동에 있어 보다 활용 가 치를 높이는 방재 지원 시스템의 구축과 함께 기상이변으로 인 한 국지성 강우를 예측하기 위해 Japan Meteorological Agency (2019)에서 제공하는 GPV(Grid point value) 데이터를 이용해 강 우-기압, 즉 태풍의 중심기압과 강우와의 연관성과 해수면 온도 와 태풍이동경로 등과 같은 주요한 학습 데이터를 구축하여 유 사태풍의 예측과 더불어 강우 사상이 포함된 통합적인 방재 시 스템 구축과 관련된 연구를 수행할 것이다.

\section{후기}

본 연구는 2017년 한국연구재단의 이공분야기초연구사업 (NRF-2017R1D1A3B03033090) 및 2018년 해양수산부(과제번호 20180404) 재원으로 해양수산과학기술진흥원의 지원을 받아 수 행된 연구결과 중 일부임을 밝히며, 연구비 지원에 감사드립니다.

\section{References}

National Institute of Informatics (NII), 2019. Digital Typhoon. [Online] Available at: $<$ http://agora.ex.nii.ac.jp/digital-typhoon/> [Accessed June 2019].

Japan Meteorological Agency (JMA), 2019. Numerical Weather Prediction Activities Service System. [Online] Available at: $<$ http://www.jma.go.jp/jma/en/Activities/nwp.html $>$ [Accessed June. 2019].

Kim, Y-J., Tanaka, K., Nakashima, H., Nakakita, E., 2015. Debris Flow Prevention Countermeasures with Urban Inundation in a Multihazard-Environment. International Journal of Erosion Control Engineering, 9(2), 58-67. https://doi.org/10.13101/ijece.9.58

Kim, Y-J., Kim, T-W, Yoon, J-S,, Kim, I-H., 2019. Study on Prediction of Similar Typhoons through Neural Network Optimization. Journal of Ocean Engineering and Technology, 33(5), 427-434. https:// doi.org/10.26748/KSOE.2019.065 
Srivastava. N., Hinton. G., Krizhevsky, A., Sutskever, I., Salakhutdinov, R., 2014. Dropout: A Simple Way to Prevent Neural Networks from Overfitting. Journal of Machine Learning Research, 15, 1929-1958.

Sugiura. M., Tsujikura. H., Tanaka. K., 2015. Interpretation on the Temporal Change of Parameters in the Flood Prediction Model based on the Reserve Function Method. Japan Society of Civil Engineers, 71(4), I_307-I_312. https://doi.org/10.2208/jscejhe. 71.I_307

Hitokoto, M., Sakuraba, M., and Sei, Y., 2016. Development of the Real-Time River Stage Predicition Method using Deep Learning.
Journal of Japan Society of Civil Engineers, Ser. B1 (Hydraulic Engineering), 72(4), I_187-I_192. https://doi.org/10.2208/jscejhe. 72.I_187

Nakatani. Y., Ishizaki. M., Nishida. S., 2017. Estimation of Water Quality Variation in a Tidal River by Applying Deep Learning Models. Journal of Japan Society of Civil Engineers, Ser. B1 (Hydraulic Engineering), 73(4), I_1141-I_1146. https://doi.org/10.2208/ jscejhe.73.I_1141

Typhoon Research center, 2019. Typhoon Information. [Online] Available at: $<$ http://www.typhoon.or.kr/> [Accessed May. 2019]. 